\title{
Circadian Rhythm and Primary Headache
}

\author{
Hee-Jin Im \\ Department of Neurology, Dongtan Sacred Heart Hospital, Hallym University Medical Center, Hwaseong, Korea
}

\begin{abstract}
The comorbidity of sleep and headache disorders has been recognized for centuries. Among various factors of sleep that interrupt or aggravate headaches_-including sleep duration, altered arousal system, sleep disordered breathing, and mood_circadian rhythm disorder can be an important factor in patients with primary headache. There is overwhelming evidence that primary headaches such as migraines and cluster headaches have periodicity and seasonal variation and are associated with chronobiological disorder through sharing a biological mechanism and the involvement of hypothalamus. This paper explores the relationship between headache and circadian rhythm. To cope with the reciprocal relationship, consideration of circadian rhythm in managing the patient with headache can reduce the severity and frequency of primary headache, leading to better quality of life for the patient.

Key Words: Sleep; Circadian rhythm; Primary headache disorders; Migraine; Chronotype

Received: March 7, 2021 Revised: April 19, 2021 Accepted: April 20, 2021

Corresponding author: Hee-Jin Im, MD, PhD, Department of Neurology, Dongtan Sacred Heart Hospital, Hallym University Medical Center, 7 Keunjaebong-gil, Hwaseong 18450, Korea.

Tel: 82-31-8680-3185, Fax: 82-31-8680-2317, E-mail: coolere@naver.com

(c) This is an Open Access article distributed under the terms of the Creative Commons Attribution Non-Commercial License (https://creativecommons.org/licenses/bync/4.0) which permits unrestricted non-commercial use, distribution, and reproduction in any medium, provided the original work is properly cited.
\end{abstract}

\section{INTRODUCTION}

Headaches are one of the most prevalent neurological disorders and most common causes of life-disabling among the general population. More than two thirds of people report headaches. The comorbidity of sleep and headache disorders has been recognized for a long time. Headaches may be both a cause and effect of sleep disturbance. Both conditions increase or worsen the risk for each other. Headaches can be a symptom of sleep disturbance and a side effect of sleep- or wake-modulating treatments. As already extensively reviewed, various sleep disorders are more prevalent among patients with primary headache than for those without headache [1]. There is general consensus and evidence, for example, of a high association between sleep breathing disorder (especially obstructive sleep apnea syndrome) and awakening headache (morning headache) [2]. Besides sleep apnea, headache may be precipitated by altered sleep patterns, such as sleep loss or oversleep among patients with migraine and tension-type headaches, the most common type of primary headache [3]. The onset, maintenance, and offset of sleep involves various mechanisms leading to a break of sleep homeostasis, periodic sleep stages [rapid eye movement (REM), or nonREM component], and circadian rhythm. Among the various factors of sleep that interrupt or aggravate headache, including sleep duration, altered arousal system, and circadian rhythm disorder can be a serious and important factor in patients with primary headache.

Several studies suggest that some headache attacks, such as in migraine and cluster headache onset, show a circadian variation and prominent circadian and circannual patterns. Chronic morning headache is associated with circadian rhythm disorder [odds ratio (OR) of 2.0], and the strength of the association was more prominent for daily morning headaches [4]. This paper explores the relationship between headaches and circadian rhythm. For clinicians treating headache patients, it is important to identify and recognize the patients' patterns of headache attack and the sleep/ wake cycle for integrated management of headache.

\section{CIRCADIAN PERIODICITY AND HEADACHE}

Every biological function is regulated by some form of circadian rhythm, most prominently demonstrated by the striking pattern of the sleep/wake cycle. This also affects our daily lives and medical disorders such as headaches. There is some evidence of the relationship between headache syndromes and cyclic phenomena. Migraines are one of the most common neurological disorders associated with a serious burden on and impairment of quality of 
life $[5,6]$. They are characterized by recurrent attacks, unilateral location, a throbbing or pulsating quality, and aggravation by routine physical activity and head movement. Migraine attacks have seasonal, menstrual, and circadian periodicity, suggesting a role of chronobiological mechanisms. Migraine periodicity was first described in 1992. More than half of migraineurs reported headaches with periodicity about four-hour blocks of time. The migraineurs showed a circadian variation in migraine onset and reported increased attack especially during the early morning, between 6 a.m. and 8 a.m., with peak frequency of headache onset between 8 a.m. and 10 a.m [7]. Another study demonstrated that migraine attacks started more frequently between $4 \mathrm{a} . \mathrm{m}$. and $9 \mathrm{a} . \mathrm{m}$. during the menstruation period [8]. This suggests that waking from sleep with a morning headache can be part of a common symptom for migraines, unless there are no other sleep disorders, such as obstructive sleep apnea. The other study also reported that attack of headache occurred on average during the period from 4 a.m. to 9 a.m. among 1,698 migraineurs [8]. A recently published article reports that fewer than half of patients with primary headache, and especially migraine and tension-type headache had the time preference of headache attack ( $45.5 \%$ in migraine, and $44.8 \%$ in tension-type headache) [9]. There was also seasonal periodicity of migraine attack frequency, not only for the 24-hour day, which was a tendency of over-presentation in the summer compared to the average seasons overall [8]. This kind of association between primary headache and seasonal preference has also been indicated for cluster headaches. Cluster headaches are an uncommon but debilitating and painful primary headache syndrome characterized by a severe, unilateral, stabbing nature, especially around orbital area [10]. Because of the distinctive features of circadian and circannual periodicity, a complex relationship also exists between chronobiology and cluster headaches. REM sleep is known to precipitate cluster headache attacks in some patients, and clusters of attack occur in specific times and seasons $[11,12]$.

\section{SHARING THE PATHOPHYSIOLOGICAL ASPECTS}

Headaches and sleep share pathophysiological mechanisms. The periodicity of migraine attacks is known to be related to hypothalamic involvement. The hypothalamus is also involved in the control of pain and in the pathogenic mechanisms of headaches. The hypothalamus, especially its posterior regions, becomes activated during attacks of the trigeminal autonomic cephalalgias, while brainstem, especially dorsal pontine, activity shows up during migraine attacks [13]. Migraines may be defined as an episodic brain disorder in which transient hypothalamic dysfunction is proposed to modulate or initiate an attack. In regard to sleep, the anterior hypothalamus contains the ventro-lateral preoptic nucleus, which is prominently active during sleep and which innervates neurons in the hypothalamus and the brainstem arousal pathways. Injury to this lesion may cause insomnia, such as among von Economo's patients [14]. The hypothalamus is probably involved in both the homeostatic control of sleep and the arousal system.

Along with the arousal system, the suprachiasmatic nucleus (SCN) is the circadian control center within the hypothalamus, receiving both direct and indirect retinal projections and mediating the entrainment of the circadian clock to light-dark cycles. Retinal ganglion cells activated by light project to SCN and adjacent thalmic nuclei [15]. The cyclic rhythms are generated and caused by feedback loops which give rise to the expression pattern in 24-hour cycles of several gene sets [16]. Seasonal rhythm of migraine attacks may support a role of $\mathrm{SCN}$ in the pathophysiology of migraines. Thalamic activation is not only related to the circadian system but is also known as a key process-ascending pathway to generate pain in migraine, cluster headaches, and trigeminovascular activation [17].

\section{CHRONOTYPE AND HEADACHE}

Chronotype is a personal circadian preference: when an individual tends to sleep and awaken. Circadian clocks entrain to light and dark differently in each individual, so that the differences between early and late chronotypes can create a negative influence on an individual's daily life [18].

The disrupted balance between the biological endogenous clock and the social clock may arise as an adaptive effort to maintain or catch up to the homeostasis leading to migraines. A recent largesample study showed that migraineurs were less likely to be a normal chronotype than the control subjects were. Migraineurs had greater prevalence of early chronotype compared with controls (OR 2.42), as well as late chronotypes (OR 1.69) [19]. Patients with migraines in the study also reported higher attack frequency, especially after changes in circadian rhythm and lower behavioral activity at unusual hours. A similar result was confirmed in an Italian study, that morning- and evening-type subjects were more prevalent among migraine patients than in the controls, and they reported lower sleep quality and higher disability [20]. In another recent study, the patient with time-preferential migraines had about three-hour earlier MSFsc (midpoint of sleep on free days, corrected for oversleep on free days) than did migraineurs without time-preferential attack of headaches. A later chronotype (evening type) has been associated with a higher headache frequency per month among migraineurs who have time-preference attack of their headache (Figure 1) [9].

On the other hand, consensus has not been established between menstrual periodicity and circadian disruption in migraines. An Italian study reports that ninety-three patients with pure menstrual migraines and menstrual-related migraines had no differences of distribution of chronotype [21].

\section{SHIFT WORKERS AND HEADACHE}

Disrupted in-nature circadian rhythm can commonly occur from extrinsic factors, such as social activity among rotating shift workers and night workers. Night or shift workers work during their biological resting phase, against the natural clock, and are forced to 

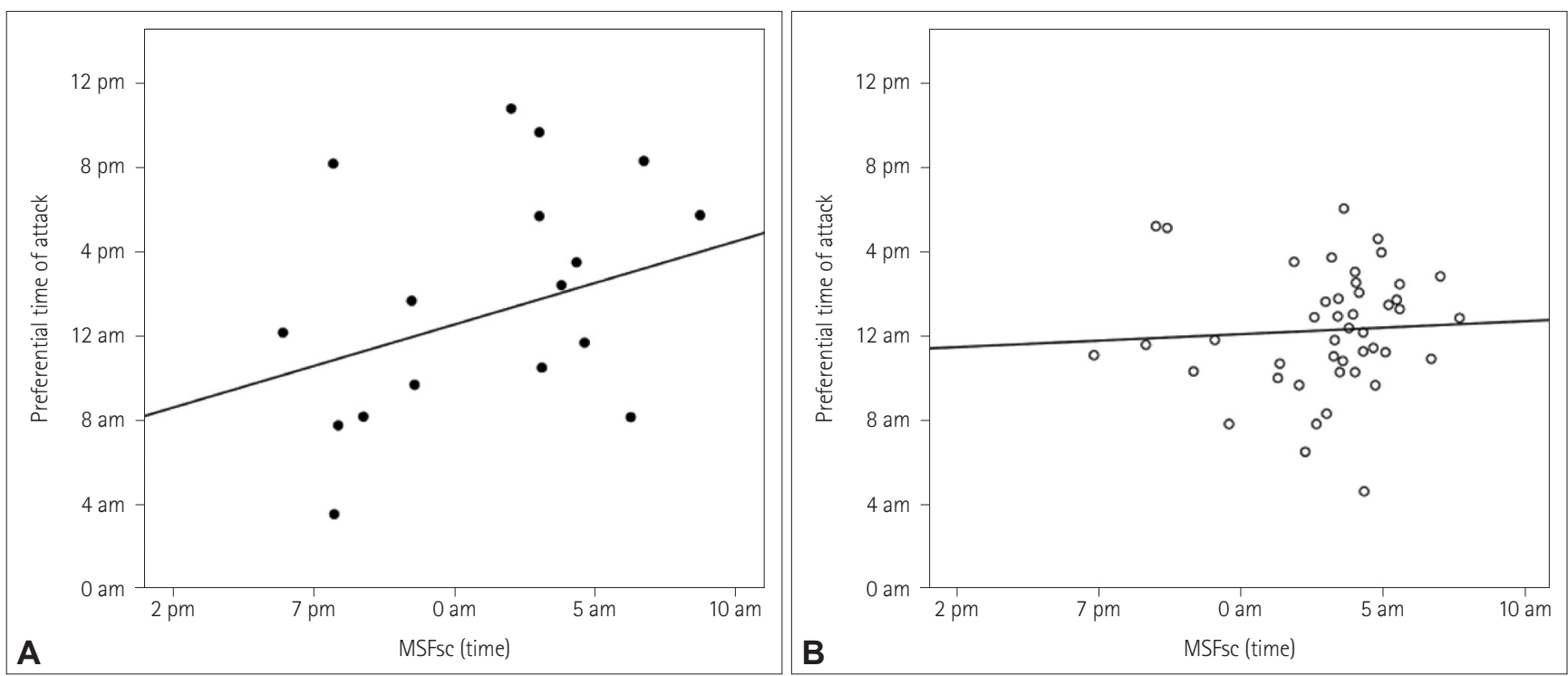

Figure 1. Association between the choronotype (MFSsc) and the preferential time of attack in patients with migraine (A) and the tension-type headache (B). MSFsc: midpoint of sleep on free days, corrected for oversleep on free days. Adapted from Im et al. Chronobiol Int 2019;36:1528-1536, with permission of Taylor \& Francis [9].

sleep during their biological active phase, with or without variation. Misalignment or moving away from the endogenous biological rhythm of shift workers is associated with medical disorders such as headaches. In a large homogenous cohort study, the frequency of migraine and chronic headaches was more prevalent among shift workers (OR 2.04; 1.60; 2.45, respectively), and there was no difference according to headache type [22]. Similarly, among nursing staff, the one-year prevalence of primary headache disorders was pretty high at $45.3 \%$ : of migraines, $14.8 \%$; of tension-type headaches, 26.2\%; and of chronic daily headaches, 2.7\% [23]. The severity of headaches and the intensity of shift or night work can be correlated. Regarding the effect of the frequency of night shifts on migraines, shift workers such as nurses who work more than eight night shifts per month had a significantly higher risk of suffering the disease (29.4\%) than those working fewer than eight night shifts per month (18.9\%) [23].

\section{CIRCADIAN CORRECTION IN MANAGEMENT OF HEADACHE}

Concerning the negative effects of interfered circadian rhythm on headaches, as mentioned so far, attempts to regain a more balanced biological rhythm can be helpful in the management of headaches. Behavioral sleep interventions such as 1) scheduling a consistent bedtime that allows for eight hours of sleep; 2) eliminating watching television or looking at the cellphone display; 3) moving supper to four or more hours before bedtime; and 4) discontinuing naps have reduced migraine frequency and intensity among migraine patients $[24,25]$. To better understand the correlation between headache attack and individual circadian rhythm, integrating a form of sleep diary and headache diary can help access and manage both the sleep and the headache issues. Melatonin, a hormone of the pineal gland, generates environmental light to a bio- logical signal, which is concerned with biological timing. Response to treatment of sleep-wake-phase disorder with melatonin has been well evidenced [26]. The most frequent circadian disorder is the delayed sleep phase syndrome, characterized by difficulty in falling asleep at the usually conventional bedtime. With melatonin treatment, migraine attack was reduced in a case of delayed sleep phase syndrome [27]. Coping with circadian rhythm and headache pathophysiological mechanisms, some reports indicate that melatonin treatment reduces headaches even in the absence of a phase disorder [28].

\section{CONCLUSION}

Recent epidemiological research focuses on the established reciprocal relationship between sleep and headache, especially in relation to circadian rhythm. Chronobiological involvement has been identified in some forms of headache, especially hypnic, cluster, and migraine headaches. Sleep regulation may play a key role in headache management. In multidisciplinary settings, clinicians need to include sleep screening strategies as part of evaluating and managing patients with headaches, especially sleep or morning headaches. By considering an individual's circadian system, pharmacological and behavioral sleep regulation strategies can reduce the severity and frequency of primary headache, leading to better quality of life for the patient.

\section{Acknowledgments}

None

\section{Conflicts of Interest}

The author has no potential conflicts of interest to disclose. 


\section{ORCID iD}

\section{Hee-Jin $\operatorname{Im}(1)$}

https://orcid.org/0000-0002-8979-6521

\section{REFERENCES}

1. Paiva T, Farinha A, Martins A, Batista A, Guilleminault C. Chronic headaches and sleep disorders. Arch Intern Med 1997;157:1701-1705.

2. Owens JA, Witmans M. Sleep problems. Curr Probl Pediatr Adolesc Health Care 2004;34:154-179.

3. Penzien DB, Rains JC, Andrew ME, Galovski T, Mohammad Y, Mosely T. Relationship of daily stress, sleep, and headache: a time series analysis [abstract]. Cephalalgia 2001;21:262-263.

4. Ohayon MM. Prevalence and risk factors of morning headaches in the general population. Arch Intern Med 2004;164:97-102.

5. Blumenfeld AM, Bloudek LM, Becker WJ, Buse DC, Varon SF, Maglinte GA, et al. Patterns of use and reasons for discontinuation of prophylactic medications for episodic migraine and chronic migraine: results from the second international burden of migraine study (IBMS-II). Headache 2013; 53:644-655.

6. Lipton RB, Bigal ME, Diamond M, Freitag F, Reed ML, Stewart WF; AMPP Advisory Group. Migraine prevalence, disease burden, and the need for preventive therapy. Neurology 2007;68:343-349.

7. Solomon GD. Circadian rhythms and migraine. Cleve Clin J Med 1992;59: 326-329.

8. Fox AW, Davis RL. Migraine chronobiology. Headache 1998;38:436-441.

9. Im HJ, Baek SH, Yun CH, Chu MK. Time preference of headache attack and chronotype in migraine and tension-type headache. Chronobiol Int 2019; 36:1528-1536.

10. Halker R, Vargas B, Dodick DW. Cluster headache: diagnosis and treatment. Semin Neurol 2010;30:175-185.

11. Della Marca G, Vollono C, Rubino M, Capuano A, Di Trapani G, Mariotti P. A sleep study in cluster headache. Cephalalgia 2006;26:290-294.

12. Barloese M, Jennum P, Knudsen S, Jensen R. Cluster headache and sleep, is there a connection? A review. Cephalalgia 2012;32:481-491.

13. Cortelli P, Pierangeli G. Hypothalamus and headaches. Neurol Sci 2007;28 Suppl 2:S198-S202.

14. Dickman MS. von Economo encephalitis. Arch Neurol 2001;58:1696-1698.
15. Moore RY, Card JP. Intergeniculate leaflet: an anatomically and functionally distinct subdivision of the lateral geniculate complex. J Comp Neurol 1994; 344:403-430

16. Bell-Pedersen D, Cassone VM, Earnest DJ, Golden SS, Hardin PE, Thomas TL, et al. Circadian rhythms from multiple oscillators: lessons from diverse organisms. Nat Rev Genet 2005;6:544-556.

17. Holland PR. Headache and sleep: shared pathophysiological mechanisms. Cephalalgia 2014;34:725-744.

18. Wittmann M, Dinich J, Merrow M, Roenneberg T. Social jetlag: misalignment of biological and social time. Chronobiol Int 2006;23:497-509.

19. van Oosterhout W, van Someren E, Schoonman GG, Louter MA, Lammers GJ, Ferrari MD, et al. Chronotypes and circadian timing in migraine. Cephalalgia 2018;38:617-625.

20. Gori S, Morelli N, Maestri M, Fabbrini M, Bonanni E, Murri L. Sleep quality, chronotypes and preferential timing of attacks in migraine without aura. J Headache Pain 2005;6:258-260.

21. Cevoli S, Nicodemo M, Grimaldi D, Leonardi L, Montagna P, Cortelli P, et al. Chronotypes in menstrual migraine: a case-control study. Neurol Sci 2010;31 Suppl 1:S163-S164.

22. Bjorvatn B, Pallesen S, Moen BE, Waage S, Kristoffersen ES. Migraine, tension-type headache and medication-overuse headache in a large population of shift working nurses: a cross-sectional study in Norway. BMJ Open 2018;8:e022403.

23. Wang Y, Xie J, Yang F, Wu S, Wang H, Zhang X, et al. The prevalence of primary headache disorders and their associated factors among nursing staff in North China. J Headache Pain 2015;16:4.

24. Rains JC. Optimizing circadian cycles and behavioral insomnia treatment in migraine. Curr Pain Headache Rep 2008;12:213-219.

25. Calhoun AH, Ford S. Behavioral sleep modification may revert transformed migraine to episodic migraine. Headache 2007;47:1178-1183.

26. Dahlitz M, Alvarez B, Vignau J, English J, Arendt J, Parkes JD. Delayed sleep phase syndrome response to melatonin. Lancet 1991;337:1121-1124.

27. Nagtegaal JE, Smits MG, Swart AC, Kerkhof GA, van der Meer YG. Melatonin-responsive headache in delayed sleep phase syndrome: preliminary observations. Headache 1998;38:303-307.

28. Gagnier JJ. The therapeutic potential of melatonin in migraines and other headache types. Altern Med Rev 2001;6:383-389. 